\title{
A NOTE ON LAMARLE FORMULA IN MINKOWSKI 3-SPACE
}

\author{
UFUK ÖZTÜRK, KAZIM İLARSLAN, ESRA BETÜL KOÇ ÖZTÜRK AND EMILIJA NEŠOVIĆ
}

\begin{abstract}
The Lamarle formula is known as a simple relation between the Gaussian curvature and the distribution parameter of a non-developable ruled surface. In this paper, we obtain the Lamarle formula of a non-developable ruled surface with pseudo null base curve and null director vector field in Minkowski 3-space. We also obtain the corresponding striction line and distribution parameter of such surface. We prove that there is no Lamarle formula when the director vector field is spacelike and its derivative is null, because the ruled surface in that case is a lightlike plane. Finally, we give some examples.
\end{abstract}

\section{Introduction}

The study of the ruled surfaces plays an important role in classical differential geometry and has many applications in computer aided-designs, projective geometry, kinematics, spatial mechanisms, etc. The ruled surface in Euclidean space $\mathbb{E}^{3}$ has a parametrization of the form $x(s, t)=\alpha(s)+t e(s)$, where $\alpha(s)$ is a regular curve and $e(s)$ is a non-zero vector field along the curve. Without loss of generality, we may assume that $e(s)$ is a unit vector field, i.e. $\|e(s)\|=1$. A regular curve $\bar{\alpha}(s)$ lying on the ruled surface $x(s, t)$ in $\mathbb{E}^{3}$ with a property $\left\langle\bar{\alpha}^{\prime}(s), e^{\prime}(s)\right\rangle=0$ is called a striction line. If $e$ and $e^{\prime}$ are the unit vector fields, then $\left\{e, e^{\prime}, e \times e^{\prime}\right\}$ is an orthonormal frame (Sannia frame [5]) in $\mathbb{E}^{3}$, so the condition $g\left(\bar{\alpha}^{\prime}, e^{\prime}\right)=0$ implies

$$
\bar{\alpha}^{\prime}(s)=\lambda(s) e(s)+D(s) e(s) \times e^{\prime}(s),
$$

where $-\lambda(s)$ and $D(s)$ are the pitch function ([4]) and the distribution parameter of the ruled surface $x(s, t)$ respectively. Hence in this case $D(s)$ is given by

$$
D(s)=\left[\bar{\alpha}^{\prime}, e, e^{\prime}\right]
$$

and it measures a component of $\bar{\alpha}^{\prime}$ perpendicular to $e \times e^{\prime}$. If the Gaussian curvature $K(s, t)$ is not identically zero on $x(s, t)$, then there exists a simple relationship between $K(s, t)$ and the distribution parameter given by

$$
K(s, t)=-\frac{D^{2}(s)}{\left(D^{2}(s)+t^{2}\right)^{2}} .
$$

Received January 15, 2018, accepted April 27, 2018.

2010 Mathematics Subject Classification. 53A05; 53B30; 53C50.

Key words and phrases. Pseudo-null curve, ruled surface, Minkowski 3-space.

Corresponding author: Ufuk Öztürk. 
Relation (1.3) is known as the Lamarle formula. It is proved in [3] that in Minkowski 3-space $E_{1}^{3}$ the Lamarle formula has different forms when the vector fields $e$ and $e^{\prime}$ are the spacelike or the timelike. Also, the distribution parameter $D(s)$ has different expressions in those cases, since the striction line lies in the spacelike or in the timelike plane [3].

In this paper, we consider two remained cases: (a) $e$ is null vector field and (b) $e$ is spacelike vector field and $e^{\prime}$ is a null vector field. In both cases, as the base curve of the ruled surface $x(s, t)$ we choose a pseudo null curve $\alpha$, whose principal normal vector field $N$ and the binormal vector field $B$ are null vector fields. In case (a), we obtain the striction line and classify such ruled surfaces as spacelike, timelike and lightlike. We also derive the distribution parameter and the corresponding Lamarle formula. In case (b), we prove that there is no Lamarle formula, because the ruled surface $x(s, t)$ in that case is a lightlike plane. Finally, we give some examples.

\section{Preliminaries}

The Minkowski 3-space $\mathbb{E}_{1}^{3}$ is the Euclidean 3-space $\mathbb{E}^{3}$ provided with the standard flat metric given by

$$
g=-d x_{1}^{2}+d x_{2}^{2}+d x_{3}^{2}
$$

where $\left(x_{1}, x_{2}, x_{3}\right)$ is a rectangular coordinate system of $\mathbb{E}_{1}^{3}$. Since $g$ is an indefinite metric, recall that a vector $v \in \mathbb{E}_{1}^{3}$ can have one of three Lorentzian causal characters: it can be spacelike if $g(v, v)>0$ or $v=0$, timelike if $g(v, v)<0$ and null (lightlike) if $g(v, v)=0$ and $v \neq 0$. In particular, the norm (length) of a vector $v$ is given by $\|v\|=\sqrt{|g(v, v)|}$ and two vectors $v$ and $w$ are said to be orthogonal, if $g(\nu, w)=0$. Next, recall that an arbitrary curve $\alpha=\alpha(s)$ in $\mathbb{E}_{1}^{3}$, can locally be spacelike, timelike or null (lightlike), if all of its velocity vectors $\alpha^{\prime}(s)$ are respectively spacelike, timelike or null (lightlike) ([7]). In particular, a spacelike curve whose the principal normal $N$ and binormal $B$ are the null vectors is called a pseudo null curve ([9]).

If $\alpha$ is a pseudo null curve in $\mathbb{E}_{1}^{3}$, the Frenet formulas have the form ([9])

$$
\left[\begin{array}{c}
T^{\prime} \\
N^{\prime} \\
B^{\prime}
\end{array}\right]=\left[\begin{array}{ccc}
0 & \kappa & 0 \\
0 & \tau & 0 \\
-\kappa & 0 & -\tau
\end{array}\right]\left[\begin{array}{c}
T \\
N \\
B
\end{array}\right],
$$

where the first curvature $\kappa$ can take only two values: $\kappa=0$ when $\alpha$ is a straight line, or $\kappa=1$ in all other cases. In particular, the following conditions hold:

$$
\begin{aligned}
& g(T, T)=1, \quad g(N, N)=g(B, B)=0, \\
& g(T, N)=g(T, B)=0, \quad g(N, B)=1 .
\end{aligned}
$$


The cross products of the frame's vectors are given by

$$
T \times N=N, N \times B=T, B \times T=B .
$$

Recall that the Lorentzian vector product of two vectors $u=\left(u_{1}, u_{2}, u_{3}\right)$ and $v=\left(v_{1}, v_{2}, v_{3}\right)$ in $\mathbb{E}_{1}^{3}$ is defined by

$$
u \times v=\left(u_{3} v_{2}-u_{2} v_{3}, u_{3} v_{1}-u_{1} v_{3}, u_{1} v_{2}-u_{2} v_{1}\right) .
$$

Definition 2.1. A surface $S$ in Minkowski 3-space is called a timelike (spacelike), if the induced metric on the surface is a Lorentzian (positive definite Riemannian) metric, i.e. if the normal on the surface is a spacelike (timelike) vector.

A spacelike or a timelike surface in Minkowski 3-space is also called a non-degenerate (non-null) surface.

Definition 2.2. A surface $S$ in Minkowski 3-space is called a lightlike (null, degenerate), if the induced metric on the surface is degenerate.

The image of the map $x: I \times \mathbb{R} \rightarrow \mathbb{E}_{1}^{3}$ defined by

$$
x(s, t)=\alpha(s)+t e(s), \quad s \in I, \quad t \in \mathbb{R}
$$

is called a ruled surface in $\mathbb{E}_{1}^{3}$, where $\alpha: I \subset \mathbb{R} \rightarrow \mathbb{E}_{1}^{3}$ is a curve and $e: I \subset \mathbb{R} \rightarrow \mathbb{E}_{1}^{3}$ is a vector field along $\alpha$. The curve $\alpha$ is called base curve (or the generating curve) and $e$ are called the director curve (or the director vector filed). The rulings of a ruled surface are the straight lines $t \rightarrow \alpha(s)+t \mathbf{e}(s)$. If the tangent plane of the ruled surface is constant along a fixed ruling, then the surface is called a developable surface. In Minkowski space $\mathbb{E}_{1}^{3}$, the examples of non-degenerate developable ruled surfaces are spacelike or timelike planes, cylinders, cones and tangent surfaces. Tangent planes of such surfaces depend on only one parameter. The examples of degenerate developable ruled surfaces in $\mathbb{E}_{1}^{3}$ are lightlike planes, cylinders, cones and tangent surfaces [8].

Definition 2.3. In Minkowski 3-space a ruled surface $S$ with parametrization (2.4) is said to be cylindrical if $e(s) \times e^{\prime}(s)=0$, and non-cylindrical if $e(s) \times e^{\prime}(s) \neq 0$.

Definition 2.4. A curve $\bar{\alpha}$ lying on the surface with parametrization (2.4) is called striction line, if it satisfies the condition $g\left(\bar{\alpha}^{\prime}, e^{\prime}\right)=0$, and its points are called central points ([2]).

The Gaussian curvature $K(s, t)$ of the non-degenerate ruled surface with parametrization (2.4) is given by ([1])

$$
K(s, t)=g(\eta, \eta) \frac{L N-M^{2}}{E G-F^{2}},
$$


where $E, F, G$ are the coefficients of the first fundamental form, $L, M, N$ are the coefficients of the second fundamental form and

$$
\eta(s, t)=\frac{x_{s} \times x_{t}}{\left\|x_{s} \times x_{t}\right\|}
$$

is the unit normal vector field on $x(s, t)$.

Definition 2.5. Non-degenerate ruled surface in Minkowski 3-space is called a non-developable surface, if its Gaussian curvature $K(s, t) \neq 0$ at each point of the surface.

\section{The Lamarle formulae for ruled surfaces with pseudo null base curve in $\mathbb{E}_{1}^{3}$}

Denote by $S$ the ruled surface in $\mathbb{E}_{1}^{3}$ with parametrization

$$
\begin{aligned}
x: I \times \mathbb{R} & \rightarrow \mathbb{E}_{1}^{3} \\
(s, t) & \rightarrow x(s, t)=\alpha(s)+t e(s),
\end{aligned}
$$

where $\alpha$ is a pseudo null base curve and $e$ is a director vector field along $\alpha$. In this section, we obtain the Lamarle formula of the ruled surface (3.1) by considering two cases: (a) $e$ is a null vector field; (b) $e$ is a spacelike vector field and its derivative $e^{\prime}$ is null. In what follows, we consider this two cases separately.

(a) $g(e, e)=0$. Then $g\left(e, e^{\prime}\right)=0$, so $e^{\prime}$ is the spacelike or the null vector field. Hence we may distinguish two subcases: (a.1) $e^{\prime}$ is the spacelike; (a.2) $e^{\prime}$ is the null.

(a.1) If $e^{\prime}(s)$ is a spacelike vector field where $s$ denotes the arc-length parameter of $e$, then $g\left(e^{\prime}(s), e^{\prime}(s)\right)=1$. In the first theorem we obtain the striction line $\bar{\alpha}$ of $S$.

Theorem 3.1. Let $S$ be the ruled surface in $\mathbb{E}_{1}^{3}$ with parametrization (3.1). Then the striction line of $S$ is given by

$$
\bar{\alpha}(s)=\alpha(s)-g\left(\alpha^{\prime}(s), e^{\prime}(s)\right) e(s) .
$$

Proof. Assume that the curve $\bar{\alpha}(s)$ lies on the ruled surface $S$. Then

$$
\bar{\alpha}(s)=\alpha(s)+u(s) e(s),
$$

where $u(s)$ is a differentiable function. Next assume that $\bar{\alpha}$ satisfies the condition $g\left(\bar{\alpha}^{\prime}, e^{\prime}\right)=0$. Differentiating the equation (3.3) with respect to $s$, we obtain

$$
\bar{\alpha}^{\prime}(s)=\alpha^{\prime}(s)+u^{\prime}(s) e(s)+u(s) e^{\prime}(s) .
$$

By taking the scalar product of the both sides of the last equation with $e^{\prime}$ and using the conditions $g\left(e, e^{\prime}\right)=g\left(\bar{\alpha}^{\prime}, e^{\prime}\right)=0$, and $g\left(e^{\prime}, e^{\prime}\right)=1$, we get

$$
u(s)=-g\left(\alpha^{\prime}(s), e^{\prime}(s)\right) .
$$


Substituting this in (3.3) we obtain (3.2).

In particular, it can be easily verified that the striction line is a unique. Differentiating the equation (3.2) with respect to $s$, we get

$$
\bar{\alpha}^{\prime}=\alpha^{\prime}-\left(g\left(\alpha^{\prime}, e^{\prime}\right)\right)^{\prime} e-g\left(\alpha^{\prime}, e^{\prime}\right) e^{\prime}
$$

Multiplying the last equation with $e$, it follows that

$$
g\left(\bar{\alpha}^{\prime}, e\right)=g\left(\alpha^{\prime}, e\right)
$$

By using (3.4), the following theorem can be proved.

Theorem 3.2. Let $S$ be the ruled surface in $\mathbb{E}_{1}^{3}$ with parametrization (3.1) and director vector field satisfying the conditions $g(e, e)=0, g\left(e^{\prime}, e^{\prime}\right)=1$. Then $S$ is a timelike surface if and only if $g\left(\bar{\alpha}^{\prime}, e\right)=g\left(\alpha^{\prime}, e\right) \neq 0$.

Proof. Assume that $S$ has the parametrization (3.1). By taking the partial derivatives of the relation (3.1) with respect to $s$ and $t$, we obtain respectively

$$
x_{s}=\alpha^{\prime}(s)+t e^{\prime}(s), \quad x_{t}=e(s) .
$$

Hence the coefficients of the first fundamental form are given by

$$
E=g\left(x_{s}, x_{s}\right), \quad F=g\left(x_{s}, x_{t}\right)=g\left(\alpha^{\prime}, e\right), \quad G=g\left(x_{t}, x_{t}\right)=0 .
$$

By using (3.4), it follows that

$$
\left(x_{s} \times x_{t}\right)^{2}=-E G+F^{2}=F^{2}=g\left(\alpha^{\prime}, e\right)^{2}=g\left(\bar{\alpha}^{\prime}, e\right)^{2} \text {. }
$$

Therefore, $S$ is a timelike surface if and only if $g\left(\alpha^{\prime}, e\right) \neq 0$.

Corollary 3.3. There is no spacelike ruled surface in $\mathbb{E}_{1}^{3}$ with the null rulings.

Corollary 3.4. If the ruled surface $S$ with null ruling in $\mathbb{E}_{1}^{3}$ is lightlike, then its striction line is null curve.

Note that when $g(e, e)=0$ and $g\left(e^{\prime}, e^{\prime}\right)=1$, then there exists a unique transversal null vector field $e^{t}$ satisfying the conditions $g\left(e, e^{t}\right)=1, g\left(e^{\prime}, e^{t}\right)=0$. Consequently, $\left\{e, e^{\prime}, e^{t}\right\}$ is an pseudo-orthonormal basis of $E_{1}^{3}$ ([6]). With respect to this basis, the tangent vector of the striction curve $\bar{\alpha}$ can be written as

$$
\bar{\alpha}^{\prime}=\lambda e+D e^{t}
$$

where the function $-\lambda=-g\left(\bar{\alpha}^{\prime}, e^{t}\right)$ is called the pitch function ([4]) and the function $D$ is called the distribution parameter. Hence the next theorem can be proved. 
Theorem 3.5. Let $S$ be the timelike ruled surface in $\mathbb{E}_{1}^{3}$ with the striction line $\bar{\alpha}$ and director vector field e satisfying the conditions $g(e, e)=0, g\left(e^{\prime}, e^{\prime}\right)=1$. Then the distribution parameter of $S$ is given by

$$
D(s)=g\left(\bar{\alpha}^{\prime}, e\right) \neq 0 .
$$

Proof. By taking the scalar product of the relation (3.5) with $e$, we get the relation (3.6). If $g\left(\bar{\alpha}^{\prime}, e\right)=0$, by using the relation (3.4) we have $g\left(\alpha^{\prime}, e\right)=0$. The last equation and the relation $g\left(e, e^{\prime}\right)=0$ imply that the spacelike vector $\alpha^{\prime}$ is collinear with the null vector $e$, which is a contradiction. Therefore, $g\left(\bar{\alpha}^{\prime}, e\right) \neq 0$.

Theorem 3.6. The Lamarle formula of the timelike ruled surface $S$ in $\mathbb{E}_{1}^{3}$ with parametrization (3.1) and director vector field e satisfying the conditions $g(e, e)=0$ and $g\left(e^{\prime}, e^{\prime}\right)=1$ reads

$$
K(s, t)=\frac{1}{D^{2}(s)}
$$

Proof. Assume that the ruled surface $S$ has the parametrization (3.1). By taking the partial derivatives of the relation (3.1) with respect to $s$ and $t$, we obtain

$$
x_{s}=\bar{\alpha}^{\prime}+t e^{\prime}, \quad x_{t}=e .
$$

By using Theorem 3.5, it follows that the coefficients of the first fundamental form are given by

$$
E=g\left(x_{s}, x_{s}\right), \quad F=g\left(x_{s}, x_{t}\right)=g\left(\bar{\alpha}^{\prime}, e\right)=D, \quad G=g\left(x_{t}, x_{t}\right)=0 .
$$

Consequently,

$$
\left(x_{s} \times x_{t}\right)^{2}=-E G+F^{2}=D^{2} .
$$

On the other hand, by taking the partial derivatives of (3.7) with respect to $s$ and $t$, we find

$$
x_{s s}=\bar{\alpha}^{\prime \prime}+t e^{\prime \prime}, \quad x_{s t}=e^{\prime}, \quad x_{t t}=0 .
$$

By using (2.6), it follows that the unit spacelike normal vector field on $S$ is given by

$$
\eta=\frac{\bar{\alpha}^{\prime} \times e+t e \times e^{\prime}}{\sqrt{D^{2}}} .
$$

Since $x_{t t}=0$, the coefficient $N=g\left(x_{t t}, \eta\right)$ of the second fundamental form is zero. Next the relations (3.8), (3.10) and (3.11) imply

$$
M=g\left(x_{s t}, \eta\right)=\frac{D}{\sqrt{D^{2}}}=\operatorname{sgn}(D) .
$$

Therefore,

$$
L N-M^{2}=-M^{2}=-1 .
$$


Finally, the relations (3.9) and (3.13) imply that the Lamarle formula has the form

$$
K(s, t)=g(\eta, \eta) \frac{L N-M^{2}}{E G-F^{2}}=\frac{1}{D^{2}(s)} .
$$

Example. Let $S$ be the ruled surface in $\mathbb{E}_{1}^{3}$ with parametrization (3.1) and pseudo null base curve given by (Figure 1)

$$
\alpha(s)=\left(e^{s}, s, e^{s}\right)
$$

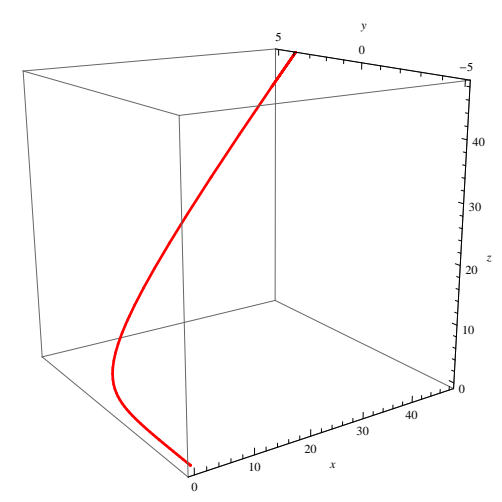

Figure 1: The pseudo null helix $\alpha$.

where $s$ is the arc-length parameter of $\alpha$.

The Frenet frame of $\alpha$ reads

$$
\begin{aligned}
& T(s)=\left(e^{s}, 1, e^{s}\right), \\
& N(s)=\left(e^{s}, 0, e^{s}\right), \\
& B(s)=\left(-\frac{e^{2 s}+1}{2 e^{s}},-1, \frac{1-e^{2 s}}{2 e^{s}}\right),
\end{aligned}
$$

Since the curvature functions of $\alpha$ are constants given by $\kappa(s)=1$ and $\tau(s)=1, \alpha$ is pseudo null helix. Assume that null director vector field $e$ of $S$ is given by

$$
e(s)=e^{-s} T(s)+\frac{e^{-2 s}}{2} N(s)-B(s) .
$$

Differentiating the last relation with respect to $s$ and using (2.1), we find

$$
e^{\prime}(s)=\left(1-e^{-s}\right) T(s)+\left(e^{-s}-\frac{e^{-2 s}}{2}\right) N(s)+B(s) .
$$

Hence $e^{\prime}$ is the unit vector field. The relations (3.1), (3.14), (3.15) and (3.16) imply that $S$ has parametrization of the form (Figure 2)

$$
x(s, t)=\left(e^{s}+t\left(e^{-s}+1+\frac{e^{s}}{2}\right), s+t\left(e^{-s}+1\right), e^{s}+t\left(1+\frac{e^{s}}{2}\right)\right) .
$$




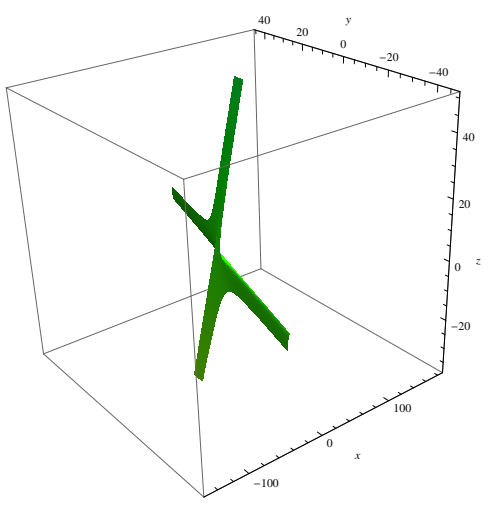

Figure 2: The timelike ruled surface $S$.

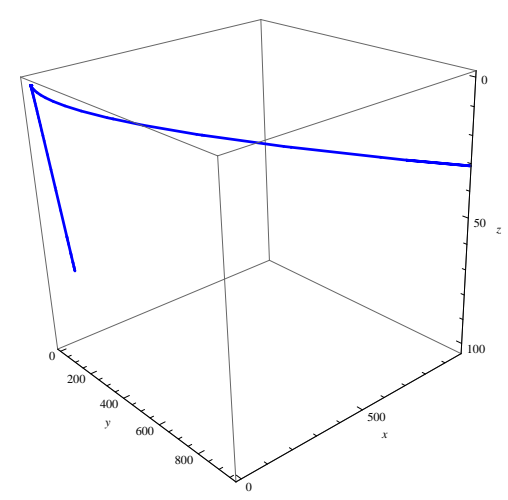

Figure 3: The striction line $\bar{\alpha}$ of $S$.

According to Theorem 3.1, the striction line $\bar{\alpha}$ of $S$ is given by (Figure 3)

$$
\bar{\alpha}(s)=\left(e^{-2 s}-\frac{1}{2}+\frac{e^{s}}{2}, s+e^{-2 s}-1, e^{-s}-\frac{1}{2}+\frac{e^{s}}{2}\right) .
$$

By using Theorems 3.5 and 3.6, we obtain the distribution parameter and the Gaussian curvature of $S$ as follows

$$
D(s)=e^{-s}, \quad K(s, t)=e^{2 s} .
$$

(a.2) If $e^{\prime}$ is null, then $e$ and $e^{\prime}$ are collinear null vectors, so the ruled surface is cylindrical ruled surface. Then the next theorem holds.

Theorem 3.7. Let $S$ be the ruled surface in $\mathbb{E}_{1}^{3}$ with parametrization (3.1), director vector field $e$ satisfying the conditions $g(e, e)=0, g\left(e^{\prime}, e^{\prime}\right)=0$ and whose base curve is striction line. Then $S$ is a lightlike cylindrical ruled surface.

By using Theorem 3.7, we conclude that in this case there is no Lamarle formula, since the ruled surface is a lightlike developable surface. 
Example. Consider the principal normal surface over pseudo null base curve $\alpha$ in $\mathbb{E}_{1}^{3}$ with parametrization

$$
x(s, t)=\alpha(s)+t N(s),
$$

where $N(s)$ is the principal normal of $\alpha$ and $s$ is the arclength parameter of $\alpha$. By using (2.1) and (2.2), we find $g\left(\alpha^{\prime}, N^{\prime}\right)=g(T, \tau N)=0$. Hence $\alpha$ is the striction line on $S$. Since $g(N, N)=$ $g\left(N^{\prime}, N^{\prime}\right)=0$, according to Theorem 3.7 the surface $S$ is a lightlike cylindrical ruled surface (Figure 4).

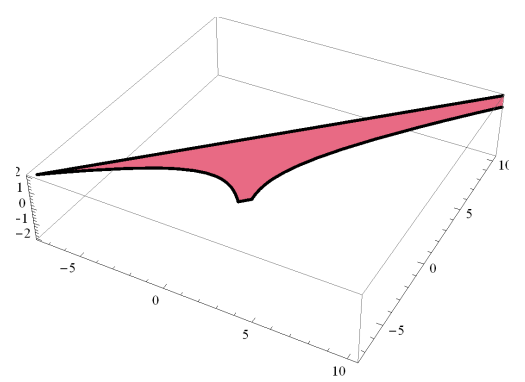

Figure 4: Principal normal surface.

(b) $e$ is a spacelike vector field and its derivative derivative $e^{\prime}$ is null. Then we may assume that $e$ is the unit spacelike vector field, i.e. $g(e, e)=1$.

Theorem 3.8. Let $\bar{S}$ be the ruled surface in $\mathbb{E}_{1}^{3}$ with the striction line $\bar{\alpha}$ and director vector field e satisfying the conditions $g(e, e)=1$ and $g\left(e^{\prime}, e^{\prime}\right)=0$. Then $\bar{S}$ is the lightlike plane of $\mathbb{E}_{1}^{3}$.

Proof. Assume that the ruled surface $\bar{S}$ has the parametrization

$$
x(s, t)=\bar{\alpha}(s)+t e(s)
$$

where $\bar{\alpha}$ is the striction line and $e$ is director vector field satisfying the conditions $g(e, e)=1$ and $g\left(e^{\prime}, e^{\prime}\right)=0$. It follows that directory curve $e(s)$ is a null curve lying in the unit pseudosphere $S_{1}^{2}(1)$. Therefore, $e$ is a null straight line. Up to isometries of $\mathbb{E}_{1}^{3}$, we may assume that $e$ has parameter equation $e(s)=(s, s, 1)$. Hence $e(s)$ lies in the lightlike plane with the equation $x_{1}=x_{2}$. Next, assume that $\bar{\alpha}$ has parameter equation $\bar{\alpha}(s)=\left(\bar{\alpha}_{1}(s), \bar{\alpha}_{2}(s), \bar{\alpha}_{3}(s)\right)$, where $\bar{\alpha}_{1}, \bar{\alpha}_{2}, \bar{\alpha}_{3}$ are some differentiable functions. The condition $g\left(\bar{\alpha}^{\prime}, e^{\prime}\right)=0$ yields $\bar{\alpha}_{1}^{\prime}=\bar{\alpha}_{2}^{\prime}$. Up to a translation of $\mathbb{E}_{1}^{3}$, we obtain that the striction line has parameter equation $\bar{\alpha}=\left(\bar{\alpha}_{1}, \bar{\alpha}_{1}, \bar{\alpha}_{3}\right)$. Consequently, the ruled surface $\bar{S}$ is the lightlike plane with the equation $x_{1}=x_{2}$.

\section{Acknowledgement}

The last author was partially supported by the Serbian Ministry of Education, Science and Technological Development (grant number 174012). 


\section{References}

[1] F. Dillen and W. Kühnel, Ruled Weingarten surfaces in Minkowski 3-space, Manuscripta Math., 98(1999), 307-320

[2] M.P. do Carmo, Differential geometry of curves and surfaces, Prentice-Hall, New Jersey, 1976.

[3] S. Ersoy and M. Tosun, Lamarle formula in 3-dimensional Lorentz space, Math. Commun., 16(2011), 593607.

[4] H. Liu and Y. Yuan, Pitch functions of ruled surfaces and B-scrolls in Minkowski 3-space, J. Geom. Phys., 62(2012), 47-52.

[5] H. Pottmann and J. Wallner, Computational Line Geometry, Springer-Verlag, Berlin, 2001.

[6] K. L. Duggal and D. H. Jin, Null Curves and Hypersurfaces of Semi-Riemannian Manifolds, World Scientific, Singapore, 2007.

[7] B. O’Neill, Semi-Riemannian Geometry With Applications to Relativity, Academic Press, New York, 1983.

[8] S. Izumiya and S. Chino, Lightlike developables in Minkowski 3-space, Demonstratio Math., XLIII (2010), 387-399.

[9] J. Walrave, Curves and surfaces in Minkowski space, ProQuest LLC, Ann Arbor, MI, Thesis (Ph.D.)-Katholieke Universiteit Leuven (Belgium), 1995.

Department Mathematics, Faculty of Science, University of Çankırı Karatekin, 18100 Çankırı, Turkey.

E-mail: uuzturk@asu.edu; ozturkufuk06@gmail.com

Department Mathematics, Faculty of Sciences and Art, University of Kırıkkale, 71450 Kırıkkale, Turkey.

E-mail: kilarslan@yahoo.com

A.H.R. Mah. Çayyolu Cad. Ada Sitesi B blok No:18/12, 18100 Çankırı, Turkey.

E-mail: ekocoztu@asu.edu, e.betul.e@gmail.com

Department of Mathematics and Informatics, Faculty of Science, University of Kragujevac, 34000 Kragujevac, Serbia.

E-mail: nesovickg@sbb.rs 